\title{
CYCLIC POLYTOPES AND THE $K$-THEORY OF TRUNCATED POLYNOMIAL ALGEBRAS
}

\author{
by Lars Hesselholt ${ }^{1}$ and Ib Madsen
}

\section{IntroduCtion}

This paper calculates the relative algebraic $K$-theory $K_{*}\left(k[x] /\left(x^{n}\right),(x)\right)$ of a truncated polynomial algebra over a perfect field $k$ of positive characteristic $p$. Since the ideal generated by $x$ is nilpotent, we can apply McCarthy's theorem: the relative algebraic $K$-theory is isomorphic to the relative topological cyclic homology, [Mc], and it is the latter groups we actually evaluate.

The result is best expressed in terms of big Witt vectors. Let $\mathbf{W}_{m}(k)$ denote the big Witt vectors in $k$ of length $m$, i.e. the multiplicative group

$$
\mathbf{W}_{m}(k)=(1+x k \llbracket x \rrbracket)^{\times} /\left(1+x^{m+1} k \llbracket x \rrbracket\right)^{\times},
$$

and recall the Verschiebung map

$$
V_{n}: \mathbf{W}_{m}(k) \rightarrow \mathbf{W}_{m n}(k)
$$

given by the substitution $V_{n}(f(x))=f\left(x^{n}\right)$. The relative $K$-theory $K\left(k[x] /\left(x^{n}\right),(x)\right)$ is given by the fibration sequence

$$
K\left(k[x] /\left(x^{n}\right),(x)\right) \rightarrow K\left(k[x] /\left(x^{n}\right)\right) \rightarrow K(k),
$$

with a corresponding exact sequence of homotopy groups

$$
0 \rightarrow K_{*}\left(k[x] /\left(x^{n}\right),(x)\right) \rightarrow K_{*}\left(k[x] /\left(x^{n}\right)\right) \rightarrow K_{*}(k) \rightarrow 0 .
$$

The groups $K_{*}(k)$ were evaluted by Quillen in [Q] when $k$ is a finite field. For a general perfect field of characteristic $p>0$ one knows that the $p$-adic $K$-groups of $k$ vanish in positive degrees by $[\mathrm{K}]$. Theorem 4.2.10 below together with McCarthy's theorem gives

Theorem A. Let $k$ be a perfect field of positive characteristic. Then

$$
K_{2 m-1}\left(k[x] /\left(x^{n}\right),(x)\right) \cong \mathbf{W}_{m n}(k) / V_{n} \mathbf{W}_{m}(k)
$$

and the groups in even degrees are zero.

The result extends calculations by Aisbett and Stienstra of $K_{3}\left(k[x] /\left(x^{n}\right),(x)\right)$. It was announced in [M], but was there stated as $K_{2 m-1}\left(k[x] /\left(x^{n}\right),(x)\right) \cong \mathbf{W}_{m n-1}(k) / V_{n} \mathbf{W}_{m-1}(k)$.

In order to evaluate the topological cyclic homology $\mathrm{TC}(A)$ one begins with the topological Hochschild spectrum $T(A)$. This is an $S^{1}$-equivariant spectrum. In the case where $A=k[x] /\left(x^{n}\right)$, standard cyclic theory leads to an equivalence of spectra

$$
T(A) \simeq_{S^{1}} T(k) \wedge N^{\mathrm{cy}}\left(\Pi_{n}\right),
$$

where $N^{\text {cy }}\left(\Pi_{n}\right)$ is the cyclic bar-construction of the pointed monoid $\Pi_{n}=\left\{0,1, x, \ldots, x^{n-1}\right\}$. Thus, for each closed subgroup $C \subset S^{1}$, the homotopy groups of the fixed sets $T(A)^{C}$ may be viewed as the $C$ equivariant homology groups $T(k)_{*}^{C}\left(N^{\text {cy }}\left(\Pi_{n}\right)\right)$. Knowledge of $\pi_{*} T(A)^{C}$ in turn determine the topological cyclic homology groups of $A$. There is an equivariant splitting

$$
N^{\mathrm{cy}}\left(\Pi_{n}\right) \cong \bigvee_{s \geqslant 0} N^{\mathrm{cy}}\left(\Pi_{n} ; s\right)
$$

\footnotetext{
${ }^{1}$ Supported in part by NSF grant
} 
where $N^{\mathrm{cy}}\left(\Pi_{n} ; 0\right)=S^{0}$ and for $s \geqslant 1$ a homeomorphism

$$
N^{\mathrm{cy}}\left(\Pi_{n} ; s\right) \cong S_{+}^{1} \wedge_{C_{s}}\left(\Delta^{s-1} / C_{s} \cdot \Delta^{s-n}\right) .
$$

Here $\Delta^{s-1}$ is the standard $s-1$ simplex with $C_{s}$-action given by cyclic permutation of the vertices, and $\Delta^{s-n} \subset \Delta^{s-1}$ is the face spanned by the first $s-n+1$ vertices. Given [HM], the main difficulty we have faced in this paper is to determine the $S^{1}$-equivariant homotopy type of the right hand side in the equality above. This is done in $\S 3$ below and is based on the concept of regular cyclic polytopes. One considers $\Delta^{s-1}$ as embedded in the regular representation $\mathbb{R} C_{s}$ by mapping the vertices to the group elements. Let $d=[(s-1) / n]$, and consider the projection

$$
\pi_{d}: \mathbb{R} C_{s} \rightarrow V_{d} ; \quad V_{d}=\mathbb{C}\left(\xi_{s}\right) \oplus \cdots \oplus \mathbb{C}\left(\xi_{s}^{d}\right) .
$$

The image of $\Delta^{s-1}$ is the regular cyclic polytope $P_{s, d}$ in $\mathbb{R}^{2 d}$. It contains $Q_{s, d}=\pi_{d}\left(C_{s} \cdot \Delta^{s-n}\right)$, so we get a map

$$
\pi_{d}: \Delta^{s-1} / C_{s} \cdot \Delta^{s-n} \rightarrow P_{s, d} / Q_{s, d}
$$

For $d n<s<(d+1) n$ it turns out that $\partial P_{s, d} \subset Q_{s, d}$ and that $0 \notin Q_{s, d}$, and we can therefore compose with the projection

$$
r: P_{s, d} / Q_{s, d} \rightarrow P_{s, d} / \partial P_{s, d}=S^{V_{d}}
$$

let $\theta_{s, n}=r \circ \pi_{d}$ denote the composite. If $s=(d+1) n$, we project instead to $V_{d+1}$, and use another projection into the join of $C_{n}$ and $S^{V_{d}}$

$$
r: P_{s, d+1} / Q_{s, d+1} \rightarrow C_{n} * S^{V_{d}} .
$$

Again, let $\theta_{s, n}=r \circ \pi_{d+1}$. The following result is proved in paragraph 3 by a mixture of geometry of cyclic polytopes and homological techniques based on results from [BAG].

Theorem B. There are $S^{1}$-equivariant equivalences

$$
S_{+}^{1} \wedge_{C_{s}}\left(\Delta^{s-1} / C_{s} \cdot \Delta^{s-n}\right) \simeq_{S^{1}} \begin{cases}S_{+}^{1} \wedge_{C_{s}} S^{V_{d}} & \text { if } d n<s<(d+1) n \\ S_{+}^{1} \wedge_{C_{s}}\left(C_{n} * S^{V_{d}}\right) & \text { if } s=(d+1) n\end{cases}
$$

given by the maps $S_{+}^{1} \wedge_{C_{s}} \theta_{s, n}$.

We conjecture that the maps $\theta_{s, n}$ are themselves $C_{s}$-equivariant equivalences, such that

$$
\Delta^{s-1} / C_{s} \cdot \Delta^{s-n} \simeq_{C_{s}} \begin{cases}S^{V_{d}} & \text {, if } d n<s<(d+1) n \\ C_{n} * S^{V_{d}} & \text {, if } s=(d+1) n\end{cases}
$$

which of course implies theorem B. If $\theta_{s, n}$ is not an equivalence its cofiber $X_{s, n}$ would be rather complicated in that the $C_{s}$-module $\tilde{H}_{*}\left(X_{s, n}\right)$ has vanishing group homology by theorem $\mathrm{B}$.

It is the simplicity of the answer in theorem B which makes possible the calculation of $T(k)_{*}^{C}\left(N^{\text {cy }}\left(\Pi_{n}\right)\right)$ and hence the proof of theorem A: $V_{d}$ is an $S^{1}$-module so $S_{+}^{1} \wedge_{C_{s}} S^{V_{d}} \cong S^{1} / C_{s+} \wedge S^{V_{d}}$ and $T(k) \wedge S^{V_{d}}$ is the $V_{d}$ th deloop of the $S^{1}$-spectrum $T(k)$, so one really only needs to calculate the equivariant spectrum homology of the circles $S^{1} / C_{s}$.

It is a pleasure to acknowledge the help we have received from other mathematicians. First, we thank T. Geisser for urging us to use the topological cyclic homology techniques to prove theorem A, which he conjectured. Second, we are grateful to L. Bærentzen for some very helpful computer calculations at an early stage. Finally, we thank V. I. Arnold, M. Boij and B. Totaro for help with the key theorem 3.1.2. We had long ago conjectured it to be true, but had grave difficulties in proving it. Arnold and Totaro sent us two independent proofs, at the very same time as M. Boij supplied us with the final details in the proof we present. 


\section{Topological Hochschild homology of truncated polynomial algebras}

2.1. This section describes the Hochschild homology and its topological extension for truncated polynomial algebras, and is mostly a recollection of results from $[\mathrm{BAG}]$ and $[\mathrm{HM}]$, but the reader is also referred to $[\mathrm{H}]$ and $[\mathrm{M}]$.

We begin with the linear case. For any ring $A$ and $A$-bimodule $M$ we write $\operatorname{HH}(A ; M)$. for the simplicial abelian group with $k$-simplices

$$
\mathrm{HH}(A ; M)_{k}=M \otimes A^{\otimes k}
$$

and simplicial structure maps

$$
\begin{aligned}
d_{i}\left(m \otimes a_{1} \ldots \otimes a_{k}\right) & =m \otimes \ldots \otimes a_{i} a_{i+1} \otimes \ldots \otimes a_{k} & & , 0 \leqslant i<k \\
& =a_{k} m \otimes a_{1} \otimes \ldots \otimes a_{k-1} & & , i=k \\
s_{i}\left(m \otimes a_{1} \ldots \otimes a_{k}\right) & =m \otimes \ldots \otimes a_{i} \otimes 1 \otimes a_{i+1} \otimes \ldots \otimes a_{k} & & , 0 \leqslant i \leqslant k .
\end{aligned}
$$

The homotopy groups of the realization $\mathrm{HH}(A ; M)$, or equivalently, the homology of the associated chain complex, are the Hochschild homology groups $\operatorname{HH}_{*}(A ; M)$. When $M=A$ with its standard $A$-bimodule structure we shorten notation to $\operatorname{HH}(A)$. . The chain complex associated with $\operatorname{HH}(A ; M)$. is derived from the two-sided bar construction by tensoring with $M$ over $A \otimes A^{\mathrm{op}}$. Therefore

$$
\mathrm{HH}_{*}(A ; M) \cong \operatorname{Tor}_{*}^{A \otimes A^{\mathrm{op}}}(A, M),
$$

provided that $A$ is flat over $\mathbb{Z}$, i.e. torsion free. When the ring $A$ is of the form $\mathbb{Z}[x] /(f(x))$, where $f(x)$ is a monic polynomial, one has the much smaller (periodic) resolution

$$
\cdots \stackrel{\Delta f / \Delta x}{\longrightarrow} A \otimes A \stackrel{\Delta x}{\longrightarrow} A \otimes A \stackrel{\Delta f / \Delta x}{\longrightarrow} A \otimes A \stackrel{\Delta x}{\longrightarrow} A \otimes A \stackrel{\mu}{\longrightarrow} A \rightarrow 0
$$

where $\Delta f=1 \otimes f(x)-f(x) \otimes 1$ and $\Delta x=1 \otimes x-x \otimes 1$. Specializing to $f(x)=x^{n}$, where $A$ is a truncated polynomial algebra, one gets for $M=A$,

$$
\mathrm{HH}_{i}\left(\mathbb{Z}[x] /\left(x^{n}\right)\right) \cong \begin{cases}\mathbb{Z}[x] /\left(x^{n}\right), & \text { if } i=0 \\ x \mathbb{Z}[x] /\left(x^{n}\right), & \text { if } i>0 \text { and even } \\ \mathbb{Z}[x] /\left(x^{n-1}\right) \oplus \mathbb{Z} / n \mathbb{Z}\left\langle x^{n-1}\right\rangle, & \text { if } i \text { is odd }\end{cases}
$$

The $k$-simplices $\mathrm{HH}\left(\mathbb{Z}[x] /\left(x^{n}\right)\right)_{k}$ has a $\mathbb{Z}$-basis consisting of $x^{i_{0}} \otimes \cdots \otimes x^{i_{k}}$. We give such a monomial the weight $w=i_{0}+\cdots+i_{k}$, and note that the weight is preserved by the face and degeneracy operators. Hence we get a direct sum decomposition of simplicial abelian groups

$$
\mathrm{HH}\left(\mathbb{Z}[x] /\left(x^{n}\right)\right) . \cong \bigoplus_{s \geqslant 0} \mathrm{HH}\left(\mathbb{Z}[x] /\left(x^{n}\right) ; s\right) .
$$

The homology of the summand $\operatorname{HH}\left(\mathbb{Z}[x] /\left(x^{n}\right) ; 0\right)$. is equal to $\mathbb{Z}$ concentrated in degree zero. To evaluate the homology of the remaining summands, we note that a chain homotopy equivalence from (2.1.1) to the two-sided bar construction must have weight $(k / 2) n$, for $k$ even, and $((k-1) / 2) n+1$, for $k$ odd, $c f$. [HM], 6.3. Hence (2.1.2) shows that the non-zero groups are

$$
\begin{array}{ll}
\mathrm{HH}_{2 d}\left(\mathbb{Z}[x] /\left(x^{n}\right) ; s\right)=\mathrm{HH}_{2 d+1}\left(\mathbb{Z}[x] /\left(x^{n}\right) ; s\right)=\mathbb{Z}, & \text { if } n \text { does not divide } s \\
\mathrm{HH}_{2 d+1}\left(\mathbb{Z}[x] /\left(x^{n}\right) ; s\right)=\mathbb{Z} / n \mathbb{Z}, & \text { if } n \text { divides } s,
\end{array}
$$

where $d$ is the integer part

$$
d=\left[\frac{s-1}{n}\right]
$$


The Boenos Aires Group in [BAG] worked out explicitely the chain homotopy equivalence from (2.1.1) to the (normalized) two-sided bar construction, following the standard procedure from e.g. [ML], p. 236. This gives us the following formulas for the cycles in the Hochschild complex which carry the homology in (2.1.4):

$$
\begin{aligned}
\iota_{2 d} & =(-1)^{d} \sum_{\substack{k_{0}+\cdots+k_{d}=s-d \\
1 \leqslant k_{i} \leqslant n-1}} x^{k_{0}} \otimes x^{k_{1}} \otimes x \otimes x^{k_{2}} \otimes x \otimes \cdots \otimes x^{k_{d}} \otimes x, \\
\iota_{2 d+1}= & (-1)^{d} \sum_{\substack{k_{0}+\cdots+k_{d}=s-d-1 \\
0 \leqslant k_{i} \leqslant n-1}} x^{k_{0}} \otimes x \otimes x^{k_{1}} \otimes x \otimes x^{k_{d}} \otimes x,
\end{aligned}
$$

We note that since $s>d n$, the lower bound on $k_{i}$ is redundant. The final result we shall need from [BAG] is the value of Connes' $B$-operator. On the chain level,

$$
B\left(\iota_{2 d}\right)=-s \iota_{2 d+1},
$$

and $B$ is zero in odd degrees.

2.2. Let $\Pi$ be a pointed monoid, i.e. a monoid with a designated base point such that the product map factors to $\mu: \Pi \wedge \Pi \rightarrow \Pi$, and let $A$ be a ring. The pointed monoid algebra is the set $A(\Pi)=A[\Pi] / A[*]$ with the ring structure induced from that on $A[\Pi]$. So in particular,

$$
A[x] /\left(x^{n}\right)=A\left(\Pi_{n}\right),
$$

where $\Pi_{n}=\left\{0,1, x, \ldots, x^{n-1}\right\}$ considered as a pointed monoid with 0 as base point and $x^{n}=0$.

The cyclic bar construction, introduced by Waldhausen, is the cyclic set $N_{.}^{\text {cy }}(\Pi)$ with $k$-simplices

$$
N_{k}^{\mathrm{cy}}(\Pi)=\Pi^{\wedge(k+1)}
$$

and the Hochschild-like structure maps

$$
\begin{aligned}
& d_{i}\left(\pi_{0} \wedge \ldots \wedge \pi_{k}\right) \quad=\pi_{0} \wedge \ldots \wedge \pi_{i} \pi_{i+1} \wedge \ldots \wedge \pi_{k} \quad, 0 \leqslant i<k \\
& =\pi_{k} \pi_{0} \wedge \pi_{1} \wedge \ldots \wedge \pi_{k-1} \quad, i=k \\
& s_{i}\left(\pi_{0} \wedge \ldots \wedge \pi_{k}\right) \quad=\pi_{0} \wedge \ldots \wedge \pi_{i} \wedge 1 \wedge \pi_{i+1} \wedge \ldots \wedge \pi_{k} \quad, 0 \leqslant i \leqslant k \\
& \tau_{k}\left(\pi_{0} \wedge \ldots \wedge \pi_{k}\right) \quad=\pi_{k} \wedge \pi_{0} \wedge \ldots \wedge \pi_{k-1} .
\end{aligned}
$$

Its geometric realization, which we denote $N^{\mathrm{cy}}(\Pi)$, has a continuous $S^{1}$-action. Moreover, the reduced chains $\mathbb{Z}\left(N_{.}^{\text {cy }}(\Pi)\right)$ and $\operatorname{HH}(\mathbb{Z}(\Pi))$. are isomorphic simplicial abelian groups, so

$$
\tilde{H}_{*}\left(N^{\mathrm{cy}}(\Pi)\right) \cong \mathrm{HH}_{*}(\mathbb{Z}(\Pi))
$$

Under this isomorphism, Connes' $B$-operator is given by the composition

$$
B: \tilde{H}_{*}\left(N^{\mathrm{cy}}(\Pi)\right) \stackrel{\left[S^{1}\right]}{\longrightarrow} \tilde{H}_{*+1}\left(S_{+}^{1} \wedge N^{\mathrm{cy}}(\Pi)\right) \stackrel{\mu_{*}}{\longrightarrow} \tilde{H}_{*+1}\left(N^{\mathrm{cy}}(\Pi)\right),
$$

where the first map is exterior multiplication by $\left[S^{1}\right] \in H_{1}\left(S^{1}\right)$, and the second map is induced from the action map, cf. [H], proposition 1.4.5.

The topological Hochschild spectrum $T(A)$ of a ring $A$, is an $S^{1}$-equivariant spectrum. We refer the reader to $[\mathrm{HM}], \S 1$, or $[\mathrm{M}], 2.3-2.4$ for the definition and to [LMS] for the notion of an equivariant spectrum. In the case of a pointed monoid algebra, we have from [HM], theorem 6.1, an equivalence of $S^{1}$-equivariant spectra

$$
T(A(\Pi)) \simeq_{S^{1}} T(A) \wedge N^{\mathrm{cy}}(\Pi)
$$

where the smash product on the right is formed in the category of $S^{1}$-equivariant spectra. 
We need to determine the $S^{1}$-space $N^{\text {cy }}\left(\Pi_{n}\right)$. First, we have a decomposition analogous to (2.1.4) of the cyclic set

$$
N_{\cdot}^{\mathrm{cy}}\left(\Pi_{n}\right)=\bigvee_{s \geqslant 0} N_{\cdot}^{\mathrm{cy}}\left(\Pi_{n} ; s\right)
$$

and the realization decomposes accordingly. The isomorphism (2.2.3) is compatible with these decompositions, so we know the reduced homology $\tilde{H}\left(N^{\mathrm{cy}}\left(\Pi_{n} ; s\right)\right)$ by (2.1.4). However, to make use of $(2.2 .4)$, we need to determine the $S^{1}$-equivariant homotopy type of $N^{\mathrm{cy}}\left(\Pi_{n} ; s\right)$. We note that $N_{\cdot}^{\mathrm{cy}}\left(\Pi_{n} ; s\right)$ as a cyclic set is generated by the single simplex

$$
x^{(s)}=x \wedge \ldots \wedge x \in N_{s-1}^{\mathrm{cy}}\left(\Pi_{n} ; s\right) .
$$

Let $\Lambda_{\text {- }}^{s-1}$ be the cyclic $(s-1)$-simplex, i.e. the cyclic set with $k$-simplices $\boldsymbol{\Lambda}([k],[s-1]), c f$. [L]. Its realization is homeomorphic to $S^{1} \times \Delta^{s-1}$. We choose the homeomorphism from [HM], 6.2, where the $C_{s^{-}}$ action induced from the $C_{s}$-action in the second factor in $\boldsymbol{\Lambda}([k],[s-1])$ becomes the diagonal action of $S^{1} \times \Delta^{s-1}$ which rotates the first factor by $2 \pi / s$ and cyclically permutes the vertices of $\Delta^{s-1}$.

Lemma 2.2.6. The characteristic map $\iota_{s}: \Lambda_{\bullet}^{s-1} \rightarrow N_{\bullet}^{\text {cy }}\left(\Pi_{n} ; s\right)$ which represents $x^{(s)}$ factors over $C_{s} \cdot \Lambda^{s-n}$ and defines an $S^{1}$-equivariant homeomorphism

$$
\left(\Lambda^{s-1} / C_{s} \cdot \Lambda^{s-n}\right) / C_{s} \cong N^{\mathrm{cy}}\left(\Pi_{n} ; s\right)
$$

Proof. The composition $\Lambda_{\bullet}^{s-n} \stackrel{\left(d^{0}\right)^{n-1}}{\longrightarrow} \Lambda_{\cdot}^{s-1} \stackrel{\iota_{s}}{\longrightarrow} N_{\bullet^{c y}}^{\text {( }}\left(\Pi_{n} ; s\right)$ is constant because $d_{0}^{n-1} x^{(s)}=x^{n} \wedge x \wedge \ldots \wedge x=0$ and therefore $\tau_{s-1}^{i} \Lambda_{\bullet}^{s-n}$ also maps to zero, for $i=0,1, \ldots, s-1$. Hence $\iota_{s}$ factors over $\Lambda_{\bullet}^{s-1} / C_{s} \cdot \Lambda_{\bullet}^{s-n}$. On the other hand, $x^{(s)}$ is invariant under cyclic permutation, so we get

$$
\left(\Lambda^{s-1} / C_{s} \cdot \Lambda^{s-n}\right) / C_{s} \rightarrow N^{c y}\left(\Pi_{n} ; s\right)
$$

This is surjective because $N_{\cdot}^{\text {cy }}\left(\Pi_{n} ; s\right)$ is generated by $x^{(s)}$, and it is injective because the only relation in $N_{.}^{\text {cy }}\left(\Pi_{n} ; s\right)$ comes from $x^{n}=\dot{0}$.

\section{Cyclic polytopes and the structure of $N^{\mathrm{cy}}\left(\Pi_{n}\right)$}

3.1. We embed $\Delta^{s-1}$ in the regular representation $\mathbb{R} C_{s}$ by letting the $i$ th vertex map to $t^{i}$, where $t \in C_{s}$ is the generator, and let $\Delta^{s-n} \subset \Delta^{s-1}$ be the inclusion onto the first $s+1-n$ vertices. The $C_{s^{-}}$action on $\mathbb{R} C_{s}$ restricts to one on $\Delta^{s-1}$ and we set

$$
C_{s} \cdot \Delta^{s-n}=\bigcup_{i=0}^{s-1} t^{i} \cdot \Delta^{s-n} \subset \Delta^{s-1} .
$$

We may then restate lemma 2.2 .6 as

$$
N^{\mathrm{cy}}\left(\Pi_{n} ; s\right) \cong S_{+}^{1} \wedge_{C_{s}}\left(\Delta^{s-1} / C_{s} \cdot \Delta^{s-n}\right),
$$

where the $S^{1}$-action on $N^{\text {cy }}\left(\Pi_{n} ; s\right)$ corresponds to the induced $S^{1}$-action on the right hand. The purpose of this paragraph is to calculate explicitely the $S^{1}$-equivariant homotopy type of the right hand side of (3.1.1).

The trigonometric moment curve in $2 d$ dimensions is

$$
x(t)=\left(e^{i t}, e^{2 i t}, \ldots, e^{d i t}\right) \in \mathbb{C}^{d}
$$

or upon identifying $\mathbb{C}^{d}$ with $\mathbb{R}^{2 d}$

$$
x(t)=(\cos t, \sin t, \cos 2 t, \sin 2 t, \ldots, \cos d t, \sin d t) \in \mathbb{R}^{2 d} .
$$


Consider the $s$ points $x_{j}=x(2 \pi j / s), j=0,1, \ldots, s-1$, on the trigometric moment curve. Their convex hull in $\mathbb{R}^{2 d}$ will be denoted $P_{s, d}$. It is the regular cyclic $s$-polytope in $2 d$-space, [G]. Note that $P_{s, 1}$ is the regular $s$-gon in the plane. If $s \leqslant 2 d+1$ then $P_{s, d}$ is an $(s-1)$-simplex.

The face structure of $P_{s, d}$ is completely known. We shall only need the structure of facets, i.e. codimension 1 faces, where one has Gale's evenness criterion: Picture the $s$ points $x_{j}$ on the circle (e.g. by projecting them onto $\left.P_{s, 1}\right)$. Then a facet of $P_{s, d}$ is the convex hull of $d$ pairs of points in the cyclic ordering, that is,

$$
F=\left\langle x_{i_{1}}, x_{i_{1}+1}, \ldots, x_{i_{d}}, x_{i_{d}+1}\right\rangle,
$$

where \langle\rangle denotes the convex hull in $\mathbb{R}^{2 d}$. Moreover, the facets are all $(2 d-1)$-simplices. The original and very readable account of this fact is $[\mathrm{C}]$. See also $[\mathrm{B}]$ and $[\mathrm{Z}]$.

The cyclic group $C_{s}$ acts on $P_{s, d}$ by cyclically permuting the vertices. This action is linear. Indeed, let $V_{d}$ denote the representation

$$
V_{d}=\mathbb{C}\left(\xi_{s}\right) \oplus \cdots \oplus \mathbb{C}\left(\xi_{s}^{d}\right) .
$$

Then the points $x_{0}, x_{1}, \ldots, x_{s-1}$ exactly form the orbit of $x_{0}=(1, \ldots, 1)$ under the action of $C_{s}$, and the action of $C_{s}$ on $V_{d}$ restricts to the $C_{s}$-action of $P_{s, d}$ which permutes the vertices.

Let $\left\langle x_{0}, \ldots, x_{k}\right\rangle$ be the convex hull of the first $(k+1)$ points $x_{\nu}=x(2 \pi \nu / s)$, and let $E$ be the convex hull

$$
E=\langle x(t) \mid 0 \leqslant t<2 \pi d /(d+1)\rangle .
$$

We note that $\left\langle x_{0}, \ldots, x_{k}\right\rangle \subset E$ when $k / s<d /(d+1)$.

Theorem 3.1.2. $E$ does not contain 0 . In particular, $0 \notin\left\langle x_{0}, \ldots, x_{k}\right\rangle$ for $k / s<d /(d+1)$.

Proof. Let $t_{0}=2 \pi / d+1$, and let $H$ be the linear subspace

$$
H=\operatorname{Span}\left\{x(0), x\left(t_{0}\right), \ldots, x\left((d-1) t_{0}\right) ; x^{\prime}\left(t_{0}\right), \ldots, x^{\prime}\left((d-1) t_{0}\right)\right\} .
$$

Since $x(0)+x\left(t_{0}\right)+\cdots+x\left(d t_{0}\right)=0$, we have $x\left(d t_{0}\right) \in H$. Consider the distance (with sign) from $H$ to $x(t)$,

$$
\delta(t)=\operatorname{det}\left(x(0), x\left(t_{0}\right), \ldots, x\left((d-1) t_{0}\right), x^{\prime}\left(t_{0}\right), \ldots, x^{\prime}\left((d-1) t_{0}\right), x(t)\right)
$$

It is non-zero, since otherwise we would have $E \subset H$, and this is impossible since $E$ is $2 d$-dimensional, and $H$ has codimension at least 1 . Now $\delta(t)$ is a trigonometric polynomial of degree $d$,

$$
\delta(t)=\sum_{k=0}^{d}\left(a_{k} \cos k t+b_{k} \sin k t\right),
$$

and therefore has at most $2 d$ roots counted with multiplicity in the interval $\left[0, d t_{0}\right] \subset[0,2 \pi)$. (Write it as a polynomial in $e^{i t}$.) But 0 and $d t_{0}$ are roots and $t_{0}, \ldots,(d-1) t_{0}$ are double roots, so these are all roots. It follows that $\delta(t)$ has constant sign on $\left[0, d t_{0}\right], \delta(t) \geqslant 0$ or $\delta(t) \leqslant 0$. Finally,

$$
E \cap H \subset\left\langle x(0), x\left(t_{0}\right), \ldots, x\left((d-1) t_{0}\right)\right\rangle .
$$

This is a facet of the $d$-simplex $\left\langle x(0), \ldots, x\left(d t_{0}\right)\right\rangle$ and therefore does not contain 0 , the barycenter.

Remark 3.1.3. The proof above is basically the same as Totaro's proof. There is a more combinatorial proof, pointed out by G. Ziegler and by B. Totaro, which runs as follows.

The combinatorial structure of $P_{s, d}$ does not depend on the choice of points $x_{j}=x(2 \pi j / s)$. The convex hull of any $s$ distinct points on the trigonometric moment curve is combinatorially equivalent to $P_{s, d}$. Let $T$ consist of $s$ points in the interval $\left[0, d t_{0}\right]$ with $\left\{0, t_{0}, \ldots, d t_{0}\right\} \subset T$ and let $P(T)=\langle x(t) \mid t \in T\rangle$. Then $P(T)$ is a cyclic polytope and Gale's evenness criterion shows that the set

$$
F=\left\langle x(0), x\left(t_{0}\right), \ldots, x\left(d t_{0}\right)\right\rangle
$$


is a face of $P(T)$. Now 0 is the barycenter of $F$, so in particular, 0 is an interior point of $F$. It follows that $0 \notin P\left(T-\left\{d t_{0}\right\}\right)$.

We recall the structure of the regular representation $\mathbb{R} C_{s}$,

$$
\mathbb{R} C_{s} \cong \mathbb{R} \oplus V_{[(s-1) / 2]}\left(\oplus \mathbb{R}_{-}\right)
$$

where the sign representation $\mathbb{R}_{-}$occurs only for even $s$. In particular, we have a projection

$$
\pi_{d}: \mathbb{R} C_{s} \rightarrow V_{d}
$$

whenever $d \leqslant[(s-1) / 2]$. By definition $P_{s, d}=\pi_{d} \Delta^{s-1}$, and we now let $Q_{s, d}=\pi_{d}\left(C_{s} \cdot \Delta^{s-n}\right)$, or

$$
Q_{s, d}=C_{s} \cdot\left\langle x_{0}, \ldots, x_{s-n}\right\rangle=\bigcup_{i=0}^{s-1}\left\langle x_{i}, x_{i+1}, \ldots, x_{i+s-n}\right\rangle
$$

If $s<(d+1) n$ then the theorem shows that $0 \notin Q_{s, d}$. Hence radial projection away from 0 gives a $C_{s}$-equivariant map

$$
r: P_{s, d} / Q_{s, d} \rightarrow P_{s, d} / \partial P_{s, d}=S^{V_{d}}
$$

to the one-point compacification of the representation $V_{d}$.

Remark 3.1.6. If $s>n d$ then $Q_{s, d}$ contains the boundary $\partial P_{s, d}$. Indeed, the boundary is a union of the facets, given by Gale's evenness criterion. A facet

$$
F=\left\langle x_{i_{1}}, x_{i_{1}+1}, \ldots, x_{i_{d}}, x_{i_{d}+1}\right\rangle
$$

is contained in $Q_{s, d}$ if there is a gap of at least $n-1$ points between some of the vertices considered as points on the circle. The worst case is then the facet

$$
F=\left\langle x_{0}, x_{1}, x_{n}, x_{n+1}, \ldots, x_{(d-1) n}, x_{(d-1) n+1}\right\rangle
$$

or any of its cyclic consequences. But the gap between $x_{(d-1) n+1}$ and $x_{0}=x_{s}$ is $s-(d-1) n-2$ which is greater than or equal to $n-1$ precisely when $s>d n$.

Suppose that $d n<s<(d+1) n$. We expect that in this case radial projection away from 0 defines an equivariant strong deformation retract of $Q_{s, d} \subset P_{s, d}$ onto the boundary $\partial P_{s, d}$, such that the map $r$ above is a $C_{s}$-equivariant homotopy equivalence. We shall not need this however.

3.2. In this section, we consider the case where $n$ does not divide $s ; d n<s<(d+1) n$. The facets of the convex polytope $P_{s, d}$ are $(2 d-1)$-dimensional simplices, so we may triangulate $P_{s, d}$ by taking cones from $x_{0}$ of the facets which do not contain $x_{0}$,

$$
P_{s, d}=\bigcup_{x_{0} \notin F} \operatorname{cone}_{x_{0}}(F) .
$$

This triangulation is closely related to the Boenos Aires formula (2.1.5). Indeed, let

$$
\begin{array}{ll}
\mathcal{X}_{s, d}=\left\{\left(k_{0}, \ldots, k_{d}\right) \mid \sum k_{i}=s-d,\right. & \left.1 \leqslant k_{i}\right\} \\
\mathcal{Y}_{s, d}=\left\{\left(k_{0}, \ldots, k_{d}\right) \mid \sum k_{i}=s-d,\right. & \left.1 \leqslant k_{i} \leqslant n-1\right\} .
\end{array}
$$

A simple calculation in $\mathrm{HH}\left(\mathbb{Z}[x] /\left(x^{n}\right)\right)$. shows that

$$
\begin{aligned}
d_{\mathbf{k}} x^{\otimes s} & =d_{2 d+1}^{k_{0}-1} d_{2 d-1}^{k_{d}-1} \ldots d_{1}^{k_{1}-1} x^{\otimes s} \\
& =x^{k_{0}} \otimes x^{k_{1}} \otimes x \otimes x^{k_{2}} \otimes x \otimes \cdots \otimes x^{k_{d}} \otimes x,
\end{aligned}
$$


such that we may rewrite $(2.1 .5)$ as

$$
\iota_{2 d}=\sum_{\mathbf{k} \in \mathcal{Y}_{s, d}} d_{\mathbf{k}} x^{\otimes s}
$$

Dually,

$$
d^{\mathbf{k}}: \Delta^{2 d} \rightarrow \Delta^{s-1} ; \quad d^{\mathbf{k}}=\left(d^{1}\right)^{k_{1}-1}\left(d^{3}\right)^{k_{2}-1} \ldots\left(d^{2 d+1}\right)^{k_{0}-1}
$$

gives a $2 d$-face of $\Delta^{s-1}$. If we embed $\Delta^{s-1}$ in $\mathbb{R} C_{s}$ as above, then $d^{\mathbf{k}}$ is the affine map given by the ordered set of vertices

$$
\left(1, t^{k_{1}}, t^{k_{1}+1}, t^{k_{1}+k_{2}+1}, t^{k_{1}+k_{2}+2}, \ldots, t^{k_{1}+\cdots+k_{d}+d-1}, t^{k_{1}+\cdots+k_{d}+d}\right) .
$$

The projection $\pi_{d}: \Delta^{s-1} \rightarrow P_{s, d}$ of (3.1.4) maps $t^{i}$ to $x_{i}$, so we get

$$
\pi_{d}\left(d^{\mathbf{k}}\left(\Delta^{2 d}\right)\right)=\text { cone }_{x_{0}}\left(F_{\mathbf{k}}\right)
$$

where $F_{\mathbf{k}}$ is the facet of $P_{s, d}=\pi_{d}\left(\Delta^{s-1}\right)$ given by

$$
F_{\mathbf{k}}=\left\langle x_{k_{1}}, x_{k_{1}+1}, x_{k_{1}+k_{2}+1}, x_{k_{1}+k_{2}+2}, \ldots, x_{k_{1}+\cdots+k_{d}+d-1}, x_{k_{1}+\cdots+k_{d}+d}\right\rangle .
$$

Conversely, by Gale's evenness criterion any facet, which does not contain $x_{0}$, has this form, and hence

$$
P_{s, d}=\bigcup_{\mathbf{k} \in \mathcal{X}_{s, d}} \operatorname{cone}_{x_{0}}\left(F_{\mathbf{k}}\right)
$$

The numbers $k_{0}-1, \ldots, k_{d}-1$ give the width of the gaps between the vertices in the simplex cone $x_{x_{0}}\left(F_{\mathbf{k}}\right)$. Hence the simplex $\pi_{d} d^{\mathbf{k}}\left(\Delta^{2 d}\right)$ is in $Q_{s, d}$ if and only if one of these gaps is at least $n-1$, or equivalently, if and only if $\mathbf{k} \in \mathcal{X}_{s, d}-\mathcal{Y}_{s, d}$.

Lemma 3.2.5. The chain $c=\sum_{\mathbf{k} \in \mathcal{Y}_{s, d}} d^{\mathbf{k}}$ is a cycle in $C_{*}\left(\Delta^{s-1}, C_{s} \cdot \Delta^{s-n}\right)$, and $r_{*} \pi_{d *}(c)$ generates $H_{2 d}\left(S^{V_{d}}\right)$.

Proof. We note that the sum $k_{i}+k_{j}$ of any pair of indices is strictly greater than $n-1$. For on the one hand $k_{0}+\cdots+k_{d}=s-d>(n-1) d$, and on the other hand each $k_{r} \leqslant n-1$, and hence

$$
k_{i}+k_{j}>(n-1) d-(n-1)(d-1)=n-1
$$

In particular, $d_{0}\left(d^{\mathbf{k}}\right) \in C_{*}\left(C_{s} \cdot \Delta^{s-n}\right)$, since the gap between $t^{k_{1}+\cdots+k_{d}+d}$ and $t^{k_{1}}$ is $k_{0}+k_{1}-1$ and hence greater than or equal to $n-1$.

Now consider $d_{1} d^{\mathbf{k}}$. If $k_{1}=n-1$ then the gap between 1 and $t^{k_{1}+1}$ is $n-1$ and hence $d_{1}\left(\left(d^{\mathbf{k}}\right) \in C_{*}\left(C_{s} \Delta^{s-n}\right)\right.$. If $k_{1} \leqslant n-2$ then the remaining $k_{i}$ are at least 2 , so we also have $\mathbf{l} \in \mathcal{Y}_{s, d}$, where

$$
\mathbf{l}= \begin{cases}\left(k_{0}, k_{1}+1, k_{2}-1, k_{3}, \ldots, k_{d}\right), & \text { if } d \geqslant 2 \\ \left(k_{0}-1, k_{1}+1\right), & \text { if } d=1\end{cases}
$$

But $d_{1}\left(d^{\mathbf{k}}\right)=d_{2}\left(d^{\mathbf{l}}\right)$ and the two faces appear with opposite sign in the boundary of the chain $c$. The other faces $d_{i}\left(d^{\mathbf{k}}\right)$ are treated analogously.

By Poincaré duality the $2 d$ th homology of $P_{s, d} / Q_{s, d}$ is free abelian of rank equal to the number of connected components of $P_{s, d}-Q_{s, d}$. The simplicial chain $\pi_{d *}(c)$ consists of simplices of $P_{s, d}$ which covers $P_{s, d}-Q_{s, d}$. Thus it represents the sum of the generators of the summands in $H_{2 d}\left(P_{s, d}, Q_{s, d}\right)$. The map $r$ maps the connected component of $P_{s, d}-Q_{s, d}$ which contains 0 to $S^{V_{d}}$ by a degree one map, and it follows that $r_{*} \pi_{d *}(c)$ generates $H_{2 d}\left(S^{V_{d}}\right)$. 
Proposition 3.2.6. The map $S_{+}^{1} \wedge_{C_{s}}\left(\Delta^{s-1} / C_{s} \Delta^{s-n}\right) \rightarrow S_{+}^{1} \wedge_{C_{s}} S^{V_{d}}$, induced from the composition of $r$ and $\pi_{d}$ is a homotopy equivalence.

Proof. Both spaces are simply connected when $d \geqslant 1$, so it suffices to argue that the map is a homology equivalence. To this end, we consider the commutative diagram

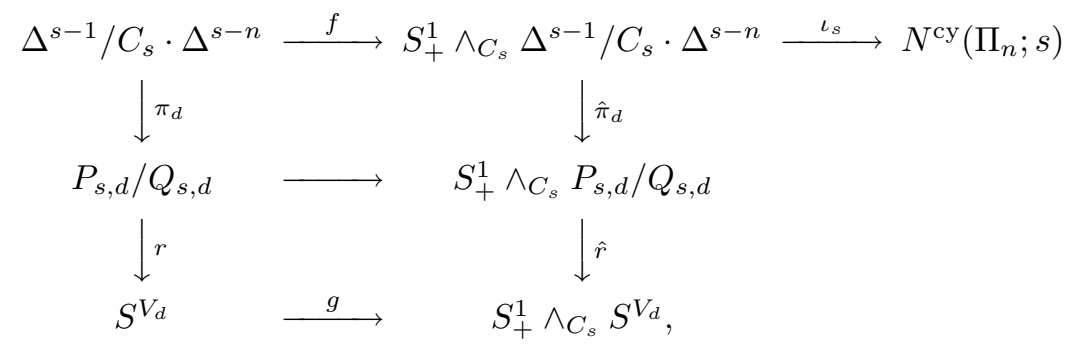

where $f$ and $g$ maps $u$ to $[1, u]$. We remarked above that $r_{*} \pi_{d *}(c)$ represents the generator. Since $V_{d}$ extends to an $S^{1}$-module,

$$
S_{+}^{1} \wedge_{C_{s}} S^{V_{d}} \cong S^{1} / C_{s+} \wedge S^{V_{d}} ; \quad[z, v] \mapsto[z, z v] .
$$

This implies that $g$ induces an isomorphism on homology in dimension $2 d$, and that the composite

$$
\tilde{H}_{2 d}\left(S_{+}^{1} \wedge_{C_{s}} S^{V_{d}}\right) \stackrel{\left[S^{1}\right]}{\longrightarrow} \tilde{H}_{2 d+1}\left(S_{+}^{1} \wedge\left(S_{+}^{1} \wedge_{C_{s}} S^{V_{d}}\right)\right) \stackrel{\mu_{*}}{\longrightarrow} \tilde{H}_{2 d+1}\left(S_{+}^{1} \wedge_{C_{s}} S^{V_{d}}\right),
$$

is multiplication by $s, c f$. (2.2.3). On the other hand, since $\iota_{s}$ is a homeomorphism by lemma 2.2.6, the Boenos Aires formula (2.1.5) and the calculation (3.2.2) shows that $f_{*}(c)$ is the generator of $H_{2 d}\left(S_{+}^{1} \wedge_{C_{s}}\right.$ $\left.\left(\Delta^{s-1} / C_{s} \cdot \Delta^{s-n}\right)\right)$. Commutativity of the diagram shows that $\hat{r} \circ \hat{\pi}_{d}$ induces an isomorphism on homology in dimension $2 d$. To prove the same in dimension $2 d+1$ we use the commutative diagram

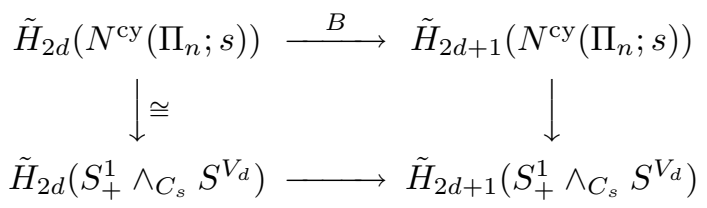

and the fact that the upper horizontal morphism is Connes' $B$-operator, hence is multiplication by $-s$ according to (2.1.6). Since the lower map multiplies by $s$, the right hand vertical map is an isomorphism.

3.3. We next treat the case where $n$ divides $s ; s=(d+1) n$ and $V_{d}=\mathbb{C}\left(\xi_{s}\right) \oplus \cdots \oplus \mathbb{C}\left(\xi_{s}^{d}\right)$. There is a cofibration sequence of $C_{s}$-spaces

$$
C_{n+} \wedge S^{V_{d}} \stackrel{\mathrm{pr}_{2}}{\longrightarrow} S^{V_{d}} \rightarrow C_{n} * S^{V_{d}}
$$

where $C_{s}$ acts via the projection onto $C_{n}$ and diagonally in the smash and join. Indeed, the mapping cone of $\mathrm{pr}_{2}$ is homeomorphic to $C_{n} * S^{V_{d}} / C_{n} * \infty$ and $C_{n} * \infty$ is contractible. Hence

$$
S_{+}^{1} \wedge_{C_{s}}\left(C_{n} * S^{V_{d}}\right) \cong\left(S^{1} \cup_{n} e^{2}\right) \wedge S^{V_{d}} .
$$

Indeed, the left hand side is the cofiber

$$
\begin{aligned}
& \operatorname{cof}\left(S_{+}^{1} \wedge_{C_{s}}\left(C_{n+} \wedge S^{V_{d}}\right) \rightarrow S_{+}^{1} \wedge_{C_{s}} S^{V_{d}}\right) \cong \operatorname{cof}\left(S_{+}^{1} \wedge_{C_{d+1}} S^{V_{d}} \rightarrow S_{+}^{1} \wedge_{C_{s}} S^{V_{d}}\right) \\
& \cong \operatorname{cof}\left(S^{1} / C_{(d+1)+} \wedge S^{V_{d}} \rightarrow S^{1} / C_{s+} \wedge S^{V_{d}}\right) \cong \operatorname{cof}\left(S^{1} / C_{(d+1)+} \rightarrow S^{1} / C_{s+}\right) \wedge S^{V_{d}} \cong\left(S^{1} \cup_{n} e^{2}\right) \wedge S^{V_{d}} .
\end{aligned}
$$

Here the second homeomorphism uses that the $C_{s}$-module $V_{d}$ is the restriction of an $S^{1}$-module so that

$$
S_{+}^{1} \wedge_{C_{s}} S^{V_{d}} \cong S^{1} / C_{s+} \wedge S^{V_{d}}, \quad[z, v] \mapsto[z, z v],
$$


where now on the right hand side the $S^{1}$-action is diagonal. In particular, $H_{2 d+1}\left(S_{+}^{1} \wedge_{C_{s}}\left(C_{n} * S^{V_{d}}\right)\right)=\mathbb{Z} / n \mathbb{Z}$. It follows from (3.3.1) that

$$
H_{2 d+1}\left(C_{n} * S^{V_{d}}\right)=I C_{n},
$$

the augmentation ideal in the integral group ring $\mathbb{Z} C_{n}$. We have

$$
I C_{n} / I C_{n}^{2} \cong C_{n}
$$

since the left hand side is $H_{0}\left(C_{n} ; I C_{n}\right)$ which is isomorphic to $H_{1}\left(C_{n} ; \mathbb{Z}\right)$ by the exact homology sequence associated with $0 \rightarrow I C_{n} \rightarrow \mathbb{Z} C_{n} \stackrel{\epsilon}{\rightarrow} \mathbb{Z} \rightarrow 0$. The isomorphism associates to $g \in C_{n}$ the class $g-1$ in $I C_{n} / I C_{n}^{2}$.

Lemma 3.3.4. The map $g: C_{n} * S^{V_{d}} \rightarrow S_{+}^{1} \wedge_{C_{s}}\left(C_{n} * S^{V_{d}}\right), g(u)=[1, u]$, induces the natural map

$$
I C_{n} \rightarrow I C_{n} / I C_{n}^{2} \cong \mathbb{Z} / n \mathbb{Z}
$$

on homology in dimension $2 d+1$.

Proof. Since the action by $C_{s}$ on $S_{+}^{1} \wedge\left(C_{n} * S^{V_{d}}\right)$ is free in the pointed sense, orbits and homotopy orbits agree, so we have a spectral sequence

$$
E^{2}=H_{*}\left(C_{s}, \tilde{H}_{*}\left(S_{+}^{1} \wedge\left(C_{n} * S^{V_{d}}\right)\right)\right) \Rightarrow \tilde{H}_{*}\left(S_{+}^{1} \wedge_{C_{s}}\left(C_{n} * S^{V_{d}}\right)\right) .
$$

It is concentrated on two horizontal lines, since

$$
\tilde{H}_{2 d+1}\left(S_{+}^{1} \wedge\left(C_{n} * S^{V_{d}}\right)\right)=\tilde{H}_{2 d+2}\left(S_{+}^{1} \wedge\left(C_{n} * S^{V_{d}}\right)\right)
$$

are the only non-zero groups. Thus both lines are $H_{*}\left(C_{s} ; I C_{n}\right)$. But

$$
H_{*}\left(C_{s} ; \mathbb{Z} C_{n}\right) \cong H_{*}\left(C_{d+1} ; \mathbb{Z}\right)
$$

by Frobenius reciprocity, $c f .[\mathrm{Br}]$, and $H_{*}\left(C_{s} ; I C_{n}\right)$ can be obtained from the exact sequence

$$
\cdots \rightarrow H_{*}\left(C_{s} ; I C_{n}\right) \rightarrow H_{*}\left(C_{s} ; \mathbb{Z} C_{n}\right) \rightarrow H_{*}\left(C_{s} ; \mathbb{Z}\right) \rightarrow \cdots
$$

It follows that $H_{*}\left(C_{s} ; I C_{n}\right)$ vanishes in odd degrees and is equal to $\mathbb{Z} / n \mathbb{Z}$ in even degrees.

By (3.3.2) the spectral sequence converges to a single copy of $\mathbb{Z} / n \mathbb{Z}$ in degree $2 d+1$. Hence all $d^{2}$ differentials must be isomorphisms, and the edge homomorphism

$$
H_{0}\left(C_{s} ; \tilde{H}_{2 d+1}\left(S_{+}^{1} \wedge\left(C_{n} * S^{V_{d}}\right)\right)\right) \rightarrow \tilde{H}_{2 d+1}\left(S_{+}^{1} \wedge_{C_{s}}\left(C_{n} * S^{V_{d}}\right)\right)
$$

is an isomorphism. It follows that the induced map $g_{*}$ of the lemma can be identified with

$$
\tilde{H}_{2 d+1}\left(C_{n} * S^{V_{d}}\right) \rightarrow H_{0}\left(C_{s} ; \tilde{H}_{2 d+1}\left(C_{n} * S^{V_{d}}\right)\right)
$$

which is precisely the map $I C_{n} \rightarrow I C_{n} / I C_{n}^{2}$.

We are now ready to determine $S_{+}^{1} \wedge_{C_{s}}\left(\Delta^{s-1} / C_{s} \cdot \Delta^{s-n}\right)$ when $s=n(d+1)$ by an argument similar to that of section 3.2. This makes use to the projection

$$
\pi_{d+1}: \Delta^{s-1} / C_{s} \cdot \Delta^{s-n} \rightarrow P_{s, d+1} / Q_{s, d+1},
$$

and of the associated $\hat{\pi}_{d+1}=S_{+}^{1} \wedge_{C_{s}} \pi_{d+1}$. We still have $0 \notin Q_{s, d+1}$, but this time $Q_{s, d+1}$ does not contain all of $\partial P_{s, d+1}$. Indeed,

$$
\begin{aligned}
\partial_{1} P_{s, d+1} & =\partial P_{s, d+1}-Q_{s, d+1} \cap \partial P_{s, d+1}=C_{n} \cdot\left\langle x_{0}, x_{1}, x_{n}, x_{n+1}, \ldots, x_{d n}, x_{d n+1}\right\rangle \\
& =\bigcup_{i=0}^{n-1}\left\langle x_{i}, x_{i+1}, \ldots, x_{i+d n}, x_{i+d n+1}\right\rangle
\end{aligned}
$$


which in turn is homotopy equivalent to a circle. This follows because each of the involved facets are joins of two $(n-1)$-simplices which each may be retracted on their barycenters, and the resulting line segments form a circle.

We write $V_{d+1}=V_{d} \oplus \mathbb{C}\left(\xi_{s}^{d+1}\right) ; \xi_{s}^{d+1}=\xi_{n}$. Then

$$
Q_{s, d+1} \cap \mathbb{C}\left(\xi_{s}^{d+1}\right)=\left\{\xi_{n}^{i} \mid i=0,1, \ldots, n-1\right\}=C_{n} \cdot\{1\}
$$

where $\mathbb{C}\left(\xi_{s}^{d+1}\right)=0 \oplus \mathbb{C}\left(\xi_{s}^{d+1}\right) \subset V_{d+1}$. Indeed, projecting to $V_{d}$ we have $0 \in\left\langle x_{0}, \ldots, x_{n d}\right\rangle \subset V_{d}$, namely,

$$
0=\frac{1}{d}\left(1+\xi_{s}^{n i}+\cdots+\xi_{s}^{n d i}\right)
$$

The proof of (3.1.2) shows that this is the only representation of 0 in the cyclic polytope $\left\langle x_{0}, \ldots, x_{n d}\right\rangle \subset V_{d}$, and (3.3.5) follows.

Let $\mathbf{n}=\left\{\frac{1}{2}\left(\xi_{n}^{i}+\xi_{n}^{i+1}\right) \mid i=0,1, \ldots, n-1\right\}$, the set of midpoints in the regular $n$-gon in $\mathbb{C}\left(\xi_{n}\right)$. Radial projection from $0 \in V_{d+1}$ gives a map

$$
r:\left(P_{s, d+1}, Q_{s, d+1}\right) \rightarrow\left(P_{s, d+1}, \partial P_{s, d+1}-\mathbf{n}\right) .
$$

On the other hand,

$$
\partial P_{s, d+1} \cong S\left(V_{d}\right) * S\left(\mathbb{C}\left(\xi_{n}\right)\right)
$$

and the general formula $X * Y-Y_{0} \simeq X *\left(Y-Y_{0}\right)$ together with the $C_{s}$-homotopy equivalence $S\left(\mathbb{C}\left(\xi_{n}\right)\right)-\mathbf{n} \simeq$ $C_{n} \cdot\{1\}$ show that

$$
\partial P_{s, d+1}-\mathbf{n} \simeq_{C_{s}} C_{n} * S\left(V_{d}\right)
$$

Thus we get a $C_{s}$-equivariant map

$$
r: P_{s, d+1} / Q_{s, d+1} \rightarrow \Sigma C_{n} * S\left(V_{d}\right) \cong C_{n} * S^{V_{d}} .
$$

This may very well be an equivariant homotopy equivalence, but we shall do with less.

The Buenos Aires formula, giving the generator $\iota_{2 d+1}$ of $\tilde{H}_{2 d+1}\left(N^{\mathrm{cy}}\left(\Pi_{n} ; s\right)\right)=\mathbb{Z} / n \mathbb{Z}$, has only one term, namely

$$
x^{n-1} \otimes x \otimes \cdots \otimes x^{n-1} \otimes x \in \mathbb{Z}\left(N_{2 d+1}^{\mathrm{cy}}\left(\Pi_{n} ; s\right)\right) .
$$

This is the iterated face $d_{2 d+2}^{n-1} d_{2 d}^{n-1} \ldots d_{2}^{n-1}$ applied to the generator $x^{\otimes s}$, so dually consider the face

$$
c: \Delta^{2 d+1} \rightarrow \Delta^{s-1} ; \quad c=\left(d^{2}\right)^{n-1} \ldots\left(d^{2 d+2}\right)^{n-1} .
$$

This is the affine map given by the ordered set of vertices

$$
\left(1, t, t^{n}, t^{n+1}, \ldots, t^{d n}, t^{d n+1}\right)
$$

in $\Delta^{s-1} \subset \mathbb{R} C_{s}$, and maps to a facet of $P_{s, d+1}$ under the projection $\pi_{d+1}$. The argument of lemma 3.2 .5 implies that $c$ becomes a cycle in $C_{*}\left(\Delta^{s-1}, C_{s} \cdot \Delta^{s-n}\right)$. Thus

$$
\pi_{d+1}(c) \in C_{2 d+1}\left(P_{s, d+1}, Q_{s, d+1}\right), \quad r \pi_{d+1}(c) \in C_{2 d+1}\left(P_{s, d+1}, \partial P_{s, d+1}-\mathbf{n}\right)
$$

are also cycles. In order to determine their homology class, we may instead determine the homology class of

$$
\partial r \pi_{d+1}(c): \partial \Delta^{2 d+1} \rightarrow \partial P_{s, d+1}-\mathbf{n} \cong S\left(V_{d+1}\right)-\mathbf{n} .
$$

We know from (3.3.4) and (3.3.6) that

$$
H_{2 d+1}\left(S\left(V_{d+1}\right)-\mathbf{n}\right)=I C_{n} .
$$

The augmentation ideal $I C_{n}$ is isomorphic to $\mathbb{Z} C_{n} /(N)$, where $N$ is the norm element. We saw above that $H_{0}\left(C_{s} ; I C_{n}\right) \cong \mathbb{Z} / n \mathbb{Z}$, and hence that $H_{0}\left(C_{s} ; \mathbb{Z} C_{n} /(N)\right) \cong \mathbb{Z} / n \mathbb{Z}$. Indeed a specific isomorphism is induced from the augmentation $\epsilon: \mathbb{Z} C_{n} /(N) \rightarrow \mathbb{Z} / n \mathbb{Z}$. 
Lemma 3.3.8. The cycle $\partial r \pi_{d+1}(c)$ represents an element of $H_{2 d}\left(S\left(V_{d+1}\right)-\mathbf{n}\right)=\mathbb{Z} C_{n} /(N)$ which augments to $1+n \mathbb{Z}$.

Proof. The boundary of $r \pi_{d+1}(c)$ is a $2 d$-dimensional sphere $S^{2 d}$ in $S^{2 d+1}=S\left(V_{d+1}\right)$ which does not intersect n. Let $\dot{D}_{+}$and $\dot{D}_{-}$be the two components of $S\left(V_{d+1}\right)-S^{2 d}$, say, $r \pi_{d+1}(c)=D_{+}$. The inclusion $S^{2 d} \subset$ $S\left(V_{d+1}\right)-\mathbf{n}$ induces an injection on homology if and only if $\mathbf{n}_{+}=D_{+} \cap \mathbf{n}$ and $\mathbf{n}_{-}=D_{-} \cap \mathbf{n}$ are both non-empty, and $\left[S^{2 d}\right] \in H_{2 d}\left(S\left(V_{d+1}\right)-\mathbf{n}\right)$ augments to $n_{+} \in \mathbb{Z} / n \mathbb{Z}, n_{+}=\# \mathbf{n}_{+}$. This follows from Poincaré duality. The dual of the homology sequence

$$
\cdots \rightarrow H_{2 d+1}\left(S\left(V_{d+1}\right)-\mathbf{n}, S^{2 d}\right) \rightarrow H_{2 d}\left(S^{2 d}\right) \rightarrow H_{2 d}\left(S\left(V_{d+1}\right)\right) \rightarrow \ldots
$$

is the sequence

$$
\cdots \rightarrow H^{0}\left(S\left(V_{d+1}\right)-S^{2 d}, \mathbf{n}\right) \rightarrow H^{1}\left(S\left(V_{d+1}\right), S\left(V_{d+1}\right)-S^{2 d}\right) \rightarrow H^{1}\left(S\left(V_{d+1}\right), \mathbf{n}\right) \rightarrow \ldots
$$

Now $S\left(V_{d+1}\right)-S^{2 d}=\dot{D}_{+} \sqcup \dot{D}_{-}$and the lower sequence is isomorphic to

$$
\cdots \rightarrow H^{0}\left(\dot{D}_{+}, \mathbf{n}_{+}\right) \oplus H^{0}\left(\dot{D}_{-}, \mathbf{n}_{-}\right) \rightarrow\left(H^{0}\left(\dot{D}_{+}\right) \oplus H^{0}\left(\dot{D}_{-}\right)\right) / \Delta \rightarrow\left(H^{0}\left(\mathbf{n}_{+}\right) \oplus H^{0}\left(\mathbf{n}_{-}\right)\right) / \Delta \rightarrow \ldots
$$

where $\Delta$ is the image of $H^{0}\left(S\left(V_{d+1}\right)\right)$. The element $(1,0) \in H^{0}\left(\dot{D}_{+}\right) \oplus H^{0}\left(\dot{D}_{-}\right)$represents the generator of $H_{2 d}\left(S^{2 d}\right)$ under duality and its image in

$$
\mathbb{Z} C_{n} /(N)=\left(H^{0}\left(\mathbf{n}_{+}\right) \oplus H^{0}\left(\mathbf{n}_{-}\right)\right) / \Delta
$$

has augmentation $\# \mathbf{n}_{+} \in \mathbb{Z} / n \mathbb{Z}$ as claimed.

We have left to check that $\mathbf{n}_{+}$consists of one point. Note that

$$
\pi_{d+1}(c)=\left\langle x_{0}, x_{1}, x_{n}, x_{n+1}, \ldots, x_{d n}, x_{d n+1}\right\rangle=\left\langle x_{0}, x_{n}, \ldots, x_{d n}\right\rangle *\left\langle x_{1}, x_{n+1}, \ldots, x_{d n+1}\right\rangle
$$

in $V_{d+1}$, and moreover,

$$
\left\langle x_{0}, x_{n}, \ldots, x_{d n}\right\rangle \cap \mathbb{C}\left(\xi_{s}^{d+1}\right)=\{1\}, \quad\left\langle x_{1}, x_{n+1}, \ldots, x_{d n+1}\right\rangle \cap \mathbb{C}\left(\xi_{s}^{d+1}\right)=\left\{\xi_{s}^{d+1}\right\} .
$$

Thus $\pi_{d+1}(c)$ is the line segment which joins 1 and $\xi_{s}^{d+1}$, and the midpoint is the only element of this line segment which also belongs to $\mathbf{n}$. This shows that $\pi_{d+1}(c) \cap \mathbb{C}\left(\xi_{s}^{d+1}\right) \cap \mathbf{n}$ consists of a single point, and $\mathbf{n} \subset \mathbb{C}\left(\xi_{s}^{d+1}\right)$ so $\pi_{d+1}(c) \cap \mathbf{n}$ is a one point space. The linear projection $r$ does not change this fact, i.e. $D_{+} \cap \mathbf{n}$ is also a one point space.

Proposition 3.3.9. For $s=n(d+1)$, the map $\hat{r} \circ \hat{\pi}_{d+1}$ gives a homotopy equivalence

$$
S_{+}^{1} \wedge_{C_{s}}\left(\Delta^{s-1} / C_{s} \cdot \Delta^{s-n}\right) \simeq S_{+}^{1} \wedge_{C_{s}}\left(C_{n} * S^{V_{d}}\right),
$$

where $V_{d}=\mathbb{C}\left(\xi_{s}\right) \oplus \cdots \oplus \mathbb{C}\left(\xi_{s}^{d}\right)$.

Proof. Consider the diagram

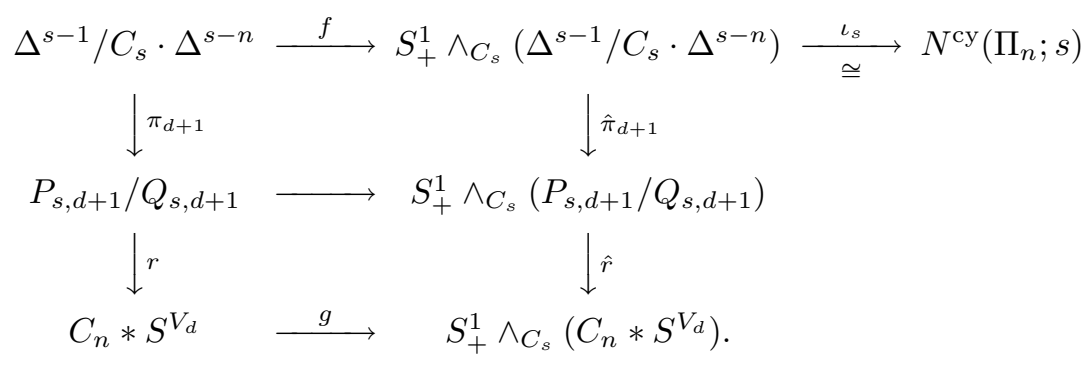

The cohomology class of the cycle $c \in C_{2 d+1}\left(\Delta^{s-1}, C_{s} \cdot \Delta^{s-n}\right)$ maps to the generator of $H_{2 d+1}\left(S_{+}^{1} \wedge_{C_{s}}\left(C_{n} *\right.\right.$ $\left.S^{V_{d}}\right)$ ) by lemmas 3.3.5 and 3.3.8. Thus $f_{*}[c]$ maps onto this generator under the right hand vertical map. 
Since $H_{*}\left(N^{\text {cy }}\left(\Pi_{n} ; s\right)\right)=\mathbb{Z} / n \mathbb{Z}$ in degree $2 d+1, \hat{r} \circ \hat{\pi}_{d+1}$ is a homology isomorphism. Both domain and target are simply connected for $d>1$. For $d=1$, a direct geometric argument gives the equivalence.

Proof of theorem B. The equivariant Whitehead theorem states that a $G$-map $f: X \rightarrow Y$ between $G$-CWcomplexes ( $G$ a compact Lie group) is a $G$-homotopy equivalence if and only if the induced map $f^{H}: X^{H} \rightarrow$ $Y^{H}$ is a weak equivalence for every closed subgroup $H \subset G$, cf. [A], [LMS]. In the case at hand, we have constructed $C_{s}$-equivariant maps

$$
\theta_{s, n}: \Delta^{s-1} / C_{s} \cdot \Delta^{s-n} \rightarrow \begin{cases}S^{V_{d}} & \text { if } d n<s<(d+1) n \\ C_{n} * S^{V_{d}} & , \text { if } s=(d+1) n\end{cases}
$$

and proved that the induced $S^{1}$-equivariant maps $S_{+}^{1} \wedge_{C_{s}} \theta_{s, n}$ are weak homotopy equivalences. Therefore, to prove theorem B we must show that the same holds on $C$-fixed sets for every closed $C \subset S^{1}$. We note that if $G$ is an abelian group, $H \subset G$ a subgroup and $X$ an $H$-space, then

$$
\left(G_{+} \wedge_{H} X\right)^{K}=\left\{\begin{array}{cl}
G / K_{+} \wedge_{H / K} X^{K} & , \text { if } K \subset H \\
* & , \text { if } K \not \subset H,
\end{array}\right.
$$

so it suffices to consider the subgroups $C_{r} \subset C_{s} \subset S^{1}$. We shall prove that in this case $\theta_{s, n}^{C_{r}}$ may be identified with $\theta_{s / r, n}$. Then theorem B follows from the equivariant Whitehead theorem.

Let $N_{r} \in \mathbb{R} C_{s}$ be the norm element of the subgroup $C_{r} \subset C_{s}$, i.e.

$$
N_{r}=1+t^{s / r}+t^{2 s / r}+\cdots+t^{(r-1) s / r}
$$

where $t \in C_{s}$ is the generator. Then

$$
\left(\Delta^{s-1}\right)^{C_{r}}=\left\langle\frac{1}{r} N_{r}, \frac{1}{r} t N_{r}, \ldots, \frac{1}{r} t^{s / r-1} N_{r}\right\rangle \cong \Delta^{s / r-1} .
$$

Moreover $\left(C_{s} \cdot \Delta^{s-n}\right)^{C_{r}}=C_{s} \cdot\left(\Delta^{s-n}\right)^{C_{r}}$ and

$$
\left(\Delta^{s-n}\right)^{C_{r}}=\left(\Delta^{s-1}\right)^{C_{r}} \cap \Delta^{s-n} \cong \Delta^{s / r-n} \subset \Delta^{s / r-1}
$$

since the representation of an element in a simplex as a convex combination of the vertices is unique. Hence

$$
\left(\Delta^{s-1} / C_{s} \cdot \Delta^{s-n}\right)^{C_{r}} \cong \Delta^{s / r-1} / C_{s / r} \cdot \Delta^{s / r-n} .
$$

One readily verifies that as $C_{r / s}$-spaces

$$
\left(S^{V_{[(s-1) / n]}}\right)^{C_{r}} \cong S^{V_{[(s / r-1) / n]}}
$$

and since $(X * Y)^{G}=X^{G} * Y^{G}$ that

$$
\left(C_{n} * S^{V_{[(s-1) / n]}}\right)^{C_{r}} \cong \begin{cases}C_{n} * S^{V_{[(s / r-1) / n]}} & , \text { if } n \text { divides } s / r \\ S^{V_{[(s / r-1) / n]}} & \text {, if } n \text { does not divide } s / r .\end{cases}
$$

Hence the maps $\theta_{s, n}^{C_{r}}$ and $\theta_{s / r, n}$ have the same domain and range, and one sees similarly that the maps are equal.

\section{TOPOLOGICAL CYCLIC HOMOLOGY OF $k[x] /\left(x^{n}\right)$}

4.1. In this paragraph, we give a general formula for the topological cyclic homology of a truncated polynomial algebra $A[x] /\left(x^{n}\right)$. We then calculate the groups $\mathrm{TC}_{*}\left(k[x] /\left(x^{n}\right)\right)$ explicitely, when the coefficient ring is a perfect field of characteristic $p>0$. First, we recall some facts about big Witt vectors and refer the reader to Bergman's lecture in $[\mathrm{Mu}]$ for details. 
Let $A$ be a commutative ring. The big Witt ring of $A$ is the set $\mathbf{W}(A)=A^{\mathbb{N}}$ equipped with a new ring structure characterized by the requirement that the ghost map

$$
w: \mathbf{W}(A) \rightarrow A^{\mathbb{N}}
$$

given by the Witt polynomials

$$
w_{n}=\sum_{d \mid n} d a_{d}^{n / d}, \quad n \geqslant 1,
$$

be a natural transformation of functors from rings to rings, when the range is given the componentwise ring structure. One has,

$$
\begin{aligned}
& \left(a_{1}, a_{2}, \ldots\right)+\left(b_{0}, b_{1}, \ldots\right)=\left(s_{1}\left(a_{1}, b_{1}\right), s_{2}\left(a_{1}, a_{2}, b_{1}, b_{2}\right), \ldots\right) \\
& \left(a_{1}, a_{2}, \ldots\right) \cdot\left(b_{0}, b_{1}, \ldots\right)=\left(p_{1}\left(a_{1}, b_{1}\right), p_{2}\left(a_{1}, a_{2}, b_{1}, b_{2}\right), \ldots\right)
\end{aligned}
$$

where $s_{n}$ and $p_{n}$ are certain polynomials, which only depend on the $a_{d}$ and $b_{d}$ where $d$ divides $n$. To see this, one proves that in the universal case $A=\mathbb{Z}\left[a_{1}, a_{2}, \ldots ; b_{1}, b_{2}, \ldots\right]$ the ghost map identifies $\mathbf{W}(A)$ with a subring of $A^{\mathbb{N}}$. The polynomials $s_{n}$ and $p_{n}$ are then defined by the formula (4.1.2), with the sum and product on the left formed in this subring structure. Since the ring axioms hold in the universal case, they hold in general.

There are operators

$$
\begin{aligned}
F_{n}: \mathbf{W}(A) \rightarrow \mathbf{W}(A), & & \text { (Frobenius) } \\
V_{n}: \mathbf{W}(A) \rightarrow \mathbf{W}(A), & & \text { (Verschiebung) }
\end{aligned}
$$

one for each $n \geqslant 1$, characterized by

$$
\begin{aligned}
F_{n}\left(w_{m}\right) & =w_{m n} \\
V_{n}\left(a_{m}\right) & = \begin{cases}a_{m / n} & \text { if } n \text { divides } m \\
0 & \text { else. }\end{cases}
\end{aligned}
$$

By the same line of reasoning as in the case of the ring structure, one shows that $F_{n}$ is well-defined and a ring homomorphism, that $V_{n}$ is addivitive and that the following formulas hold

$$
\begin{aligned}
& V_{n}\left(F_{n}(x) y\right)=x V_{n}(y) \\
& F_{n} V_{n}=n, \quad V_{n} F_{n}=\operatorname{mult}_{V_{n}(1)} \\
& F_{n} V_{m}=V_{m} F_{n}, \quad \text { if }(m, n)=1 .
\end{aligned}
$$

As noted above, the polynomials $s_{n}$ and $p_{n}$ depend only on the $a_{d}$ and $b_{d}$, where $d$ divides $n$. We can therefore, in the above discussion, replace $\mathbb{N}$ by any subset $S \subset \mathbb{N}$ which is stable under division and get a ring $\mathbf{W}_{S}(A)$. We call $\mathbf{W}_{S}(A)$ the truncated ring of big Witt vectors and $S$ the truncation set. For example, we have the ring of big Witt vectors of length $m$ defined by

$$
\mathbf{W}_{m}(A)=\mathbf{W}_{\{1,2, \ldots, m\}}(A) \text {. }
$$

Evidently, if $S \subset S^{\prime}$ are two truncation sets, then the projection $\mathbf{W}_{S^{\prime}}(A) \rightarrow \mathbf{W}_{S}(A)$ is a ring homomorphism, and moreover, the Frobenius and Verschiebung maps restrict to operators

$$
\begin{aligned}
& F_{n}: \mathbf{W}_{S}(A) \rightarrow \mathbf{W}_{S / n}(A), \\
& V_{n}: \mathbf{W}_{S / n}(A) \rightarrow \mathbf{W}_{S}(A),
\end{aligned}
$$

where $S / n$ is the truncation set $S / n=\{m \in \mathbb{N} \mid n m \in S\}$. When $S$ is the empty set, it is understood that $\mathbf{W}_{S}(A)$ is the trivial ring. 
Now let $p$ be a prime. The ring of $p$-typical Witt vectors is defined as the truncated Witt ring

$$
W(A)=\mathbf{W}_{\left\{1, p, p^{2}, \ldots\right\}}(A) .
$$

If $A$ is a $\mathbb{Z}_{(p)}$-algebra, one has a ring isomorphism

$$
\mathbf{W}(A) \cong \prod_{(d, p)=1} W(A)
$$

which on the $d$ th factor is given by the composite ring map

$$
I_{d}: \mathbf{W}(A) \stackrel{F_{d}}{\longrightarrow} \mathbf{W}(A) \stackrel{\mathrm{pr}}{\longrightarrow} W(A)
$$

see $[\mathrm{Mu}]$. More generally, we have the ring of $p$-typical Witt vectors of length $s$,

$$
W_{s}(A)=\mathbf{W}_{\left\{1, p, \ldots, p^{s-1}\right\}}(A),
$$

and, when $A$ is a $\mathbb{Z}_{(p)}$-algebra, a ring isomorphism

$$
\mathbf{W}_{m}(A) \cong \prod_{(d, p)=1} W_{s}(A), \quad p^{s-1} d \leqslant m<p^{s} d
$$

whose $d$ th factor is the composite

$$
I_{d}: \mathbf{W}_{m}(A) \stackrel{F_{d}}{\longrightarrow} \mathbf{W}_{[m / d]}(A) \stackrel{\mathrm{pr}}{\longrightarrow} W_{s}(A)
$$

Suppose that $n=p^{i} k$ with $(p, k)=1$ and let $d=e k$. Then one readily shows that the formulas (4.1.4) give a commutative square

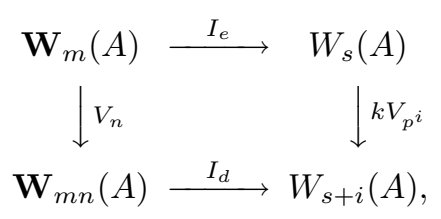

which describes the Verschiebung map under the splitting in (4.1.10).

Finally, we recall that the underlying additive group of the ring $\mathbf{W}(A)$ is isomorphic to the multiplicative group of power series in $A$ with constant term 1,

$$
\mathbf{W}(A) \cong(1+X A \llbracket X \rrbracket)^{\times}, \quad\left(a_{1}, a_{2}, \ldots\right) \mapsto \prod_{i \geqslant 1}\left(1-a_{i} X^{i}\right),
$$

and under this isomorphism

$$
F_{n}(f(X))=\prod_{i=1}^{n} f\left(\xi_{i}\right), \quad V_{n}(f(X))=f\left(X^{n}\right),
$$

where $\xi_{1}, \ldots, \xi_{n}$ are the formal $n$th roots of $X$. Similarly,

$$
\mathbf{W}_{m}(A) \cong(1+X A \llbracket X \rrbracket)^{\times} /\left(1+X^{m+1} A \llbracket X \rrbracket\right)^{\times}
$$


4.2. Given a ring $A$ and a two-sided ideal $I$, we let $T(A, I)$ denote the relative topological Hochschild spectrum, that is, the homotopy fiber of the map $T(A) \rightarrow T(A / I)$. We recall the equivalence of $S^{1}$-spectra

$$
T\left(A[x] /\left(x^{n}\right)\right) \simeq_{S^{1}} T(A) \wedge N^{\mathrm{cy}}\left(\Pi_{n}\right)
$$

where the smash product on the right is formed in the category of $S^{1}$-spectra. Thus for $C \subset S^{1}$,

$$
\pi_{*}\left(T\left(A[x] /\left(x^{n}\right)\right)^{C}\right) \cong T(A)_{*}^{C}\left(N^{\mathrm{cy}}\left(\Pi_{n}\right)\right),
$$

the $C$-equivariant homology of the $S^{1}$-space $N^{\text {cy }}\left(\Pi_{n}\right)$ with respect to the $S^{1}$-spectrum $T(A)$. The $S^{1}$-space $N^{\text {cy }}\left(\Pi_{n}\right)$ splits equivariantly as a wedge

$$
N^{\mathrm{cy}}\left(\Pi_{n}\right)=\bigvee_{s \geqslant 0} N^{\mathrm{cy}}\left(\Pi_{n} ; s\right)
$$

and the summand $N^{\text {cy }}\left(\Pi_{n} ; 0\right)=S^{0}$ corresponds to the copy of $T(A)$ in $T\left(A[x] /\left(x^{n}\right)\right)$. The equivariant homotopy type of the remaining summands is given by theorem B which we proved in $\S 3$. Let again $V_{d}$ denote the representation $V_{d}=\mathbb{C}(\xi) \oplus \cdots \oplus \mathbb{C}\left(\xi^{d}\right)$ and let $[x]$ denote the largest integer less than or equal to $x$. Then we have a cofibration sequence of $S^{1}$-spectra

$$
\bigvee_{s \geqslant 1, n \mid s} T(A)_{V_{\left[\frac{s-1}{n}\right]}} \wedge S^{1} / C_{s / n+} \stackrel{\mathrm{pr}}{\longrightarrow} \bigvee_{s \geqslant 1} T(A)_{V_{\left[\frac{s-1}{n}\right]}} \wedge S^{1} / C_{s+} \rightarrow T\left(A[x] /\left(x^{n}\right),(x)\right)
$$

Indeed, for any finite dimensional $S^{1}$-representation $V, T(A) \wedge S^{V} \simeq{ }_{S^{1}} T(A)_{V}$, the $V$ th deloop of the $S^{1}$-spectrum $T(A), c f$. [LMS], theorem I.7.9, and (4.2.1) follows from theorem B.

Recall from $[\mathrm{HM}], \S 1$, that $T(A)$ is a cyclotomic spectrum so that we have restriction maps

$$
R_{r}: T(A)_{V}^{C_{r s}} \rightarrow T(A)_{\rho_{C_{r}}^{*} V^{C_{r}}}^{C_{s}}
$$

where $\rho_{C_{r}}^{*} V^{C_{r}}$ indicates $V^{C_{r}}$ considered as an $S^{1}$-representation through the $r$ th root isomorphism $\rho_{C_{r}}: S^{1} \rightarrow$ $S^{1} / C_{r}$. In our case

$$
\rho_{C_{r}}^{*} V_{\left[\frac{r s-1}{n}\right]}^{C_{r}} \cong V_{\left[\frac{s-1}{n}\right]}
$$

such that the restriction maps are

$$
R_{r}: T(A)_{V_{\left[\frac{r s-1}{n}\right]}^{C_{r s}}}^{C_{n}} \rightarrow T(A)_{V_{\left[\frac{s-1}{n}\right]}^{C_{s}}}^{C_{s}}
$$

We can then repeat the argument of $[\mathrm{HM}], \S 8$, to get

Proposition 4.2.3. After profinite completion there is a cofibration sequence of spectra

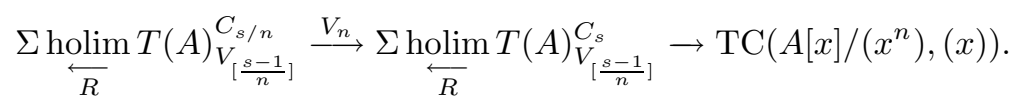

The homotopy limits runs over the natural numbers ordered by division and $T(A)_{V_{\left[\frac{s-1}{n}\right]}}^{C_{s / n}}$ is understood to be
the trivial spectrum when $n$ does not divide $s$.

By cofinality, the restriction of the homotopy limit on the left in (4.2.3) to the natural numbers divisible by $n$ gives an equivalence

$$
\underset{\leftarrow}{\operatorname{holim}} T(A)_{V_{\left[\frac{s-1}{n}\right]}^{C_{s / n}}}^{\stackrel{\sim}{\longrightarrow}} \underset{\leftarrow}{\operatorname{holim}} T(A)_{V_{r-1}}^{C_{r}}
$$


We now fix a prime $p$ and consider, for every $d$ with $(d, p)=1$, the composition

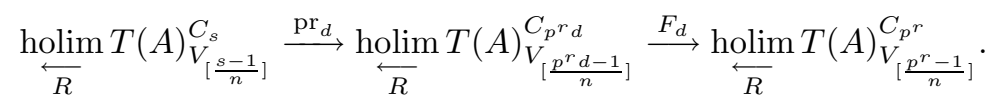

The first map is the restriction of the homotopy limit to the natural numbers of the form $p^{r} d, r \geqslant 0$, and the second map is the map of homotopy limits induced from the inclusion map

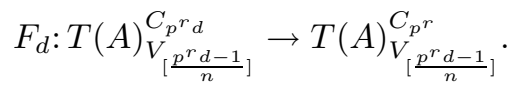

We then have the following analog of (4.1.8).

Proposition 4.2.5. Suppose that $A$ is a commutative $\mathbb{Z}_{(p)}$-algebra. Then the map

$$
\underset{R}{\operatorname{holim}} T(A)_{V_{\left[\frac{s-1}{n}\right]}^{C_{s}}}^{\stackrel{\sim}{\longrightarrow}} \prod_{(d, p)=1} \underset{R}{\operatorname{holim}} T(A)_{V_{\left[\frac{p^{r} d-1}{n}\right]}^{C_{p^{r}}}}
$$

whose $d$ th factor is $F_{d} \circ \mathrm{pr}_{d}$, is an equivalence.

Proof. Let $\mathcal{F}$ be the category associated with the poset of natural numbers ordered after division, and let $\mathcal{F}_{p}$ and $\mathcal{F}^{\prime}$ be the full subcategories of natural numbers which are powers of $p$ and prime to $p$, respectively. Then the homotopy limit on the left in (4.2.5) is indexed by $\mathcal{F}$ and the homotopy limits on the right are indexed by $\mathcal{F}_{p}$. As categories $\mathcal{F}=\mathcal{F}_{p} \times \mathcal{F}^{\prime}$ and hence we have a homeomorphism

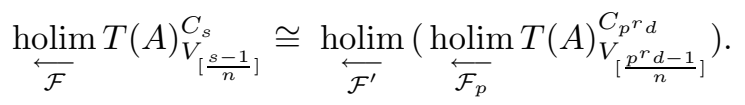

We claim that the map

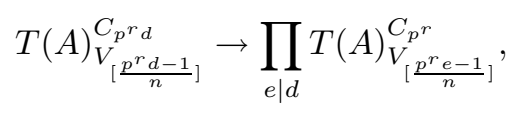

which on the eth factor is given by $R_{d / e} F_{e}$, is an equivalence. Given this, the proposition follows. To prove the claim we need the following generalization of [HM], theorem 2.2: let $T$ be a cyclotomic spectrum, let $q$ be a prime and let $l$ be a natural number prime to $q$. Then there is a cofibration sequence of (non-equivariant) spectra

$$
\left(\rho_{C_{l}}^{\#} T_{V}^{C_{l}}\right)_{h C_{q}^{i}} \stackrel{N}{\longrightarrow} T_{V}^{C_{q^{i} l}} \stackrel{R}{\longrightarrow} T_{\rho_{C_{q}}^{*} V^{C_{q}}}^{C_{q^{i-1} l}}
$$

If $V=0$ this is because $T^{C_{l}}$ is a $q$-cyclotomic spectrum. In general, it follows from loc. cit., proposition 2.1 and the proof of proposition 2.3. We write $d=q_{1}^{i_{1}} \ldots q_{m}^{i_{m}}$, where $q_{1}, \ldots, q_{m}$ are primes. Then (4.2.6) gives a cofibration sequence

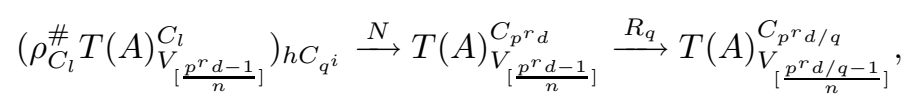

where, for notational reasons, we have written $q$ for $q_{m}, i$ for $i_{m}$ and $l$ for $p^{r} d / q^{i}$. Moreover, we have a map of cofibration sequences

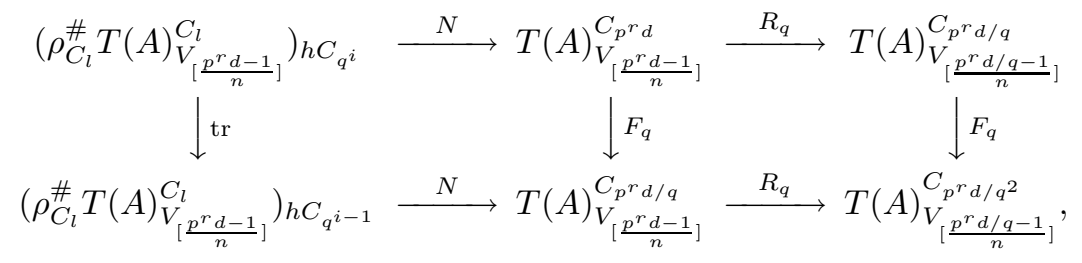


where the left hand vertical map is the transfer of the projection going in the opposite direction. We shall prove that $\operatorname{tr}$ is an equivalence such that the square on the right is homotopy cartesian. The claim then readily follows by induction on $m$ and $i_{k}, k=1, \ldots, m$.

Let us write $V$ for $V_{\left[\left(p^{r} d / q-1\right) / n\right]}$. We note that multiplication by $q$ on $\pi_{*} T(A)_{V}^{C_{l}}$ is an isomorphism. For $T(A)_{V}^{C_{l}}$ is a module spectrum over the ring spectrum $T(A)^{C_{l}}$. In particular, the homotopy groups $\pi_{*} T(A)_{V}^{C_{l}}$ are modules over the ring $\pi_{0} T(A)^{C_{l}}$. By [HM], addendum 3.3, this ring is isomorphic to the truncated big Witt ring $\mathbf{W}_{\langle l\rangle}(A),\langle l\rangle=\{d \mid d$ divides $l\}$, and $q$ is a unit in $\mathbf{W}_{\langle l\rangle}(A)$. Now, for any $S^{1}$-spectrum $T$, one has a first quadrant homology type spectral sequence

$$
E_{s, t}^{2}=H_{s}\left(C_{q^{i}} ; \pi_{t} T\right) \Rightarrow \pi_{s+t}\left(T_{h C_{q^{i}}}\right)
$$

where the homotopy groups $\pi_{*} T$ are trivial $C_{q^{i}}$-modules because the $C_{q^{i}}$-action extends to an $S^{1}$-action. The edge homomorphism of the spectral sequence is the map on homotopy groups induced from the projection $T \rightarrow T_{h C_{q^{i}}}$. When multiplication by $q$ is an isomorphism on $\pi_{*} T$, the spectral sequence collapses onto the axis $s=0$, and the edge homomorphism is an isomorphism. This shows that the projection $T_{h C_{q^{i-1}}} \rightarrow T_{h C_{q^{i}}}$ is an equivalence. But the composition

$$
T_{h C_{q^{i}}} \stackrel{\text { tr }}{\longrightarrow} T_{h C_{q^{i-1}}} \stackrel{\mathrm{pr}}{\longrightarrow} T_{h C_{q^{i}}}
$$

induces multiplication by $q$ on homotopy groups and is therefore also an equivalence. Hence tr is an equivalence. This finishes the proof.

We note that the individual terms in the homotopy limits on the right hand side of (4.2.5) approximate the limit. More precisely, the fundamental cofibration sequence, $[\mathrm{HM}]$, theorem 2.2, and the fact that taking homotopy orbits preserves connectivity, shows that the projection

$$
\underset{R}{\operatorname{holim}} T(A)_{V_{\left[\frac{p^{r} d-1}{n}\right]}^{C_{p^{r}}}}^{C_{[}} \rightarrow T(A)_{V_{\left[\frac{p^{s} d-1}{n}\right]}^{C_{p^{s}}}}
$$

is $2\left[\left(p^{s+1} d-1\right) / n\right]$-connected. We also note that the proposition gives a splitting of the left hand spectrum in (4.2.3). Indeed, if we write $n=p^{i} k$ with $(k, p)=1$, then (4.2.4) and (4.2.5) show that the map

$$
\underset{R}{\operatorname{holim}} T(A)_{V_{\left[\frac{s-1}{n}\right]}^{C_{s / n}}}^{\stackrel{\sim}{\longrightarrow}} \prod_{(e, p)=1} \underset{R}{\operatorname{holim}} T(A)_{V_{\left[\frac{p^{r} e k-1}{n}\right]}^{C_{p^{r-i}}}}^{\longleftarrow}
$$

which on the eth factor is given by $F_{e} \circ \mathrm{pr}_{e k}$, is an equivalence.

Addendum 4.2.9. Suppose that $n=p^{i} k$ with $(p, k)=1$ and let $d=e k$. Then there is a commutative diagram

$$
\begin{aligned}
& \pi_{*} \underset{R}{\operatorname{holim}} T(A)_{V_{\left[\frac{s-1}{n}\right]}^{C_{s / n}}}^{F_{e} \circ \mathrm{pr}_{d}} \longrightarrow \pi_{*} \underset{R}{\operatorname{holim}} T(A)_{V_{\left[\frac{p^{r} d-1}{n}\right]}^{C_{p^{r-i}}}}^{V_{R}} \\
& \downarrow V_{n}
\end{aligned}
$$

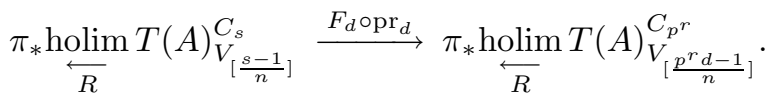

Proof. By naturality, we have a commutative square

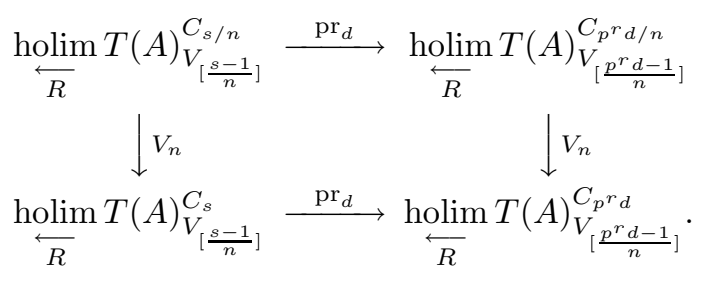


We recall from $[\mathrm{HM}]$, lemma 2.3.1, that on the level of homotopy groups $F_{s} V_{r}=V_{r} F_{s}$, provided that $(r, s)=1$. Since we have

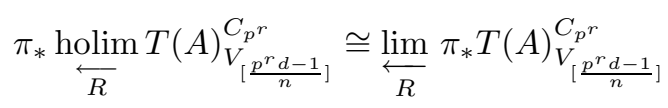

from (4.2.7), we get a commutative square

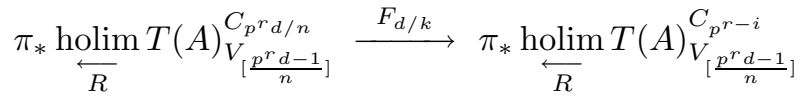

$$
\begin{aligned}
& \downarrow V_{p^{i}} \quad \downarrow V_{p^{i}}
\end{aligned}
$$

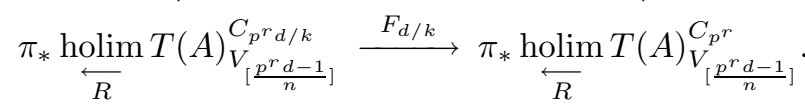

Finally, we can write $F_{d} \circ V_{n}$ as the composite

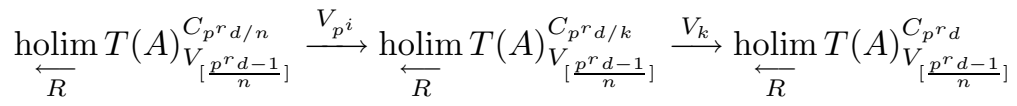

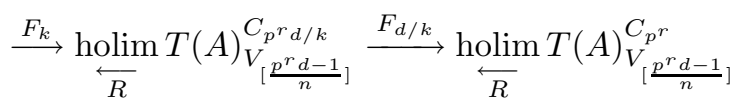

and $F_{k} \circ V_{k}$ induces multiplication by $k$ on homotopy groups.

We now assume that the coefficient ring is a perfect field $k$ of positive characteristic $p$ and proceed to prove theorem $\mathrm{A}$ of the introduction.

Theorem 4.2.10. Let $k$ be a perfect field of characteristic $p>0$. Then the long exact homotopy sequence associated with the cofibration sequence of (4.2.3) takes the form

$$
0 \rightarrow \mathbf{W}_{m}(k) \stackrel{V_{n}}{\longrightarrow} \mathbf{W}_{m n}(k) \rightarrow \mathrm{TC}_{2 m-1}\left(k[x] /\left(x^{n}\right),(x)\right) \rightarrow 0,
$$

the groups in even degrees being zero.

Proof. We have from [HM], proposition 9.1, and (4.2.7) that

$$
\pi_{2 j} \underset{R}{\operatorname{holim}} T(k)_{V_{\left[\frac{p^{r} d-1}{n}\right]}^{C_{p^{r}}}}^{\overleftarrow{n}_{n}} \cong W_{s}(k)
$$

with $s$ determined by the inequalities

$$
\left[\left(p^{s-1} d-1\right) / n\right] \leqslant j<\left[\left(p^{s} d-1\right) / n\right] .
$$

One readily checks that these inequalities are equivalent to the inequalities

$$
p^{s-1} d \leqslant n(j+1)<p^{s} d,
$$

which appear in the decomposition in (4.1.10). Therefore, by (4.2.5)

$$
\pi_{2 j} \underset{R}{\operatorname{holim}} T(k)_{V_{\left[\frac{s-1}{n}\right]}^{C_{s}}} \cong \mathbf{W}_{n(j+1)}(k)
$$

and the groups in odd degrees are zero. Similarly, we have

$$
\pi_{2 j} \underset{R}{\operatorname{holim}} T(k)_{V_{\left[\frac{p^{r} d-1}{n}\right]}^{C_{p^{r-i}}}}^{\longleftarrow} \cong W_{s-i}(k)
$$


with $s$ determined by the same inequalities as above. Suppose that $d=e k$ and let $r=s-i$. Then one immediately shows that $r$ is given by the inequalities

$$
p^{r-1} e \leqslant j+1<p^{r} e
$$

and hence

$$
\pi_{2 j} \underset{R}{\operatorname{holim}} T(k)_{V_{\left[\frac{s-1}{n}\right]}^{C_{s / n}} \cong \mathbf{W}_{j+1}(k)}
$$

Finally, we recall from $[\mathrm{HM}]$, proposition 9.1, that under the isomorphism of (4.2.11) and (4.2.12) the map

$$
V_{p^{i}}: \underset{R}{\operatorname{holim}} T(k)_{V_{\left[\frac{p^{r} d-1}{n}\right]}^{C_{p^{r-i}}}}^{\stackrel{4}{\operatorname{holim}}} T(k)_{V_{\left[\frac{p^{r} d-1}{n}\right]}^{C_{p^{r-i}}}}^{\overbrace{R}}
$$

induces the Verschiebung $V_{p^{i}}: W_{s-i}(k) \rightarrow W_{s}(k)$ of Witt vectors. Now the theorem follows from (4.1.11) and (4.2.9).

\section{REFERENCES}

[A] J. F. Adams, Prerequisites (on equivariant theory) for Carlson's lecture, LNM 1051, Springer- Verlag (1984), 483-532.

[BAG] J. A. Guccione, J. J. Guccione, M. J. Redondo, A. Solotar, O. E. Villamayor, Cyclic homology of algebras with one generator, $K$-theory 5 (1991), 51-68.

[B] A. Brøndsted, An introduction to convex polytopes, GTM 90, Springer-Verlag 1983.

[BHM] M. Bökstedt, W. C. Hsiang, I. Madsen, The cyclotomic trace and algebraic K-theory of spaces, Invent. Math. 111 (1993), 465-540.

[Br] K. Brown, Cohomology of groups, GTM 87, Springer-Verlag.

[C] C. Carathéodory, Über den Variabilitätsbereich der Fourierschen Konstanten von positiven harmonischen Funktionen, Rend. Circ. Mat. Palermo 32 (1911), 193-217.

[G] D. Gale, Neighborly and cyclic polytopes, Proc. Symp. Pure Math. 7 (1963), 225-232.

$[\mathrm{H}] \quad$ L. Hesselholt, On the p-typical curves in Quillen's K-theory, Acta Math. (to appear).

$[\mathrm{HM}] \quad$ L. Hesselholt, I. Madsen, On the K-theory of finite algebras over Witt vectors of perfect fields, Topology (to appear).

[K] C. Kratzer, $\lambda$-structure en K-théorie algébrique, Comment. Math. Helv. 55 (1980), 233-254.

[LMS] L. G. Lewis, J. P. May, M. Steinberger, Equivariant stable homotopy theory, LNM 1213, Springer- Verlag, 1986.

[L] J.-L. Loday, Cyclic homology, Grundlehren der math. Wissenschaften 301, Springer-Verlag, 1992.

[ML] S. MacLane, Homology, Grundlehren der math. Wissenschaften 114, Springer-Verlag, 1963.

[M] I. Madsen, Algebraic K-theory and traces, in Current Developments in Mathematics 1995, International Press.

[Mc] R. McCarthy, Relative algebraic K-theory and topological cyclic homology, preprint, 1996.

$[\mathrm{Mu}]$ D. Mumford, Lectures on curves on an algebraic surface, Annals of Math. Studies 59, Princeton University Press, 1966.

[Q] D. Quillen, On the cohomology and K-theory of the general linear groups over a finite field, Ann. Math. 96 (1972), $552-586$.

[Z] G. M. Ziegler, Lectures on polytopes, GTM 152, Springer-Verlag 1995.

Massachusetts Institute of Technology

Cambridge, Massachusetts

\author{
Aarhus University \\ Aarhus, Denmark
}

